CASE REPORT

\title{
Massive haemoptysis after living donor liver transplantation
}

\author{
P Aseni, M Vertemati, E Minola, E Bonacina
}

J Clin Pathol 2003;56:876-878

\begin{abstract}
A 27 year old man with hereditary haemorrhagic telangiectasia who developed progressive liver dysfunction underwent living related right lobe transplantation. Pulmonary arteriography did not reveal arteriovenous malformation or abnormal intrapulmonary venous channels. The postoperative course was characterised by persistent hypoxaemia and respiratory failure developed. On day 6, a massive haemoptysis developed and the patient died shortly thereafter. The native liver showed a nodular pseudocirrhotic transformation, with highly dilated and irregularly interconnected vein-like or arterial-like structures in the fibrous septa. Pathological examination of both lungs showed irregular thickening of the wall of the arteries, secondary to eccentric and/or concentric myointimal hyperplasia. This case suggests that massive haemoptysis can develop even when arteriovenous malformations are undetectable by pulmonary arteriography, and it questions the role and the appropriateness of living donor liver transplantation in high risk patients.
\end{abstract}

$\mathrm{H}$ ereditary haemorrhagic telangiectasia (HHT) is an autosomal dominant transmitted disorder, characterised by angiodysplastic lesions involving the nasopharynx and oropharynx, gastrointestinal system, and bronchial tree. $^{12}$

The classic triad is recurrent epistaxis, mucocutaneous telangiectasia, and hereditary transmission. ${ }^{3}$

Hepatic involvement is a rare manifestation of the disease and its prevalence ranges from $8 \%$ to $31 \%{ }^{45}$ The most frequent hepatic lesions are sinusoidal dilatation, fibrosis or cirrhosis, hepatic artery aneurysms, and arterial-venous or arterial-portal fistulas. Intrahepatic vascular shunts may lead to right heart insufficiency or failure, as a consequence of pulmonary hypertension.

Patients affected by HHT with liver involvement and left to right intrahepatic shunt complicated by pulmonary hypertension and right sided heart failure can be successfully treated by liver transplantation. ${ }^{6}$

\section{CASE REPORT}

A 27 year old man was admitted to the hospital for consideration of liver transplantation with a diagnosis of cryptogenic cirrhosis and hepatopulmonary syndrome.

The patient was well until the age of 18, when he was submitted to brachial artery embolisation of an arteriovenous malformation of the right elbow. He had repeated episodes of epistaxis.

In February 1998, the patient developed rapidly worsening dyspnoea, which needed treatment with oxygen (pulmonary arterial pressure, $21 / 6 \mathrm{mmHg}$ ).

In October 1999, pulmonary and hepatic arteriography did not reveal arteriovenous malformations in either the lungs or the liver.

In May 2001, liver function tests and clinical conditions quickly deteriorated (bilirubin, $4.6 \mathrm{mg} / \mathrm{litre}$; international normalised ratio, 1.6; albumin, $23 \mathrm{~g} / \mathrm{litre}$; and platelets, $55.000 / \mathrm{mm}^{3}$ ) and he was referred to our hospital. The patient was in severe respiratory distress and he required treatment with oxygen. The liver, which was tender and not pulsatile, extended $3 \mathrm{~cm}$ below the right costal margin. Two vascular malformations ( $2 \mathrm{~cm}$ in diameter) were noted on the skin covering the nose. The splenic tip was not palpable.

An arterial blood sample showed hypoxaemia at rest (partial oxygen pressure, $50 \mathrm{mmHg}$ ). Orthodeoxya was absent. The diffusion capacity was $16 \mathrm{ml} / \mathrm{minute} / \mathrm{mmHg}$ ( $59 \%$ of predicted).

The patient was negative for hepatitis B surface antigen, hepatitis C virus RNA, and anti-human immunodeficiency virus antibodies.

HHT was diagnosed because of the presence of two symptoms of the classic triad; that is, cutaneous vascular malformation and recurrent epistaxis. Inheritance was doubtful.

Although a review of previous pulmonary arteriography did not show evidence of direct right to left shunts, the pronounced dyspnoea with severe hypoxaemia, in the absence of interstitial lung disease or pulmonary hypertension, were thought possibly to be related to microscopic arteriovenous malformations. In June 2001, the patient was submitted to living related (mother) right liver transplantation.

The immediate postoperative course was characterised by persistent hypoxaemia. On day 2, the patient had an episode of nasal and oropharynx bleeding. Fibre optic examination showed telangiectasia in the nasal cavity and bleeding from the left lower turbinate. On day 3, his clinical condition worsened. A chest $x$ ray showed bilateral pulmonary atelectasia and pulmonary oedema. Fibre optic bronchoscopy revealed blood clots in the left main bronchus.

On day 6, a massive haemoptysis developed and the patient died shortly thereafter.

Pathological examination of the native liver showed a grossly nodular surface (nodules ranged in size from 0.5 to $3 \mathrm{~cm}$ ), with small spider-like vessels visible on the glissonian capsule. The portal vein was extremely dilated, without thrombi.

Histological examination of the liver parenchyma showed a nodular pseudocirrhotic transformation, owing to the presence of extremely large fibrous septa irregularly dividing the parenchyma into large near normal "macrolobules" containing numerous normal portal tracts and central veins (fig 1). In the fibrous septa, irregular blood vessels could be recognised, forming an "archipelago" encased in the fibrous tissue, in which dilated vein-like or arterial-like structures were present and irregularly interconnected. Some of these vessels entered the parenchyma, simulating the features of hepatoportal sclerosis (fig 1B).

Postmortem examination of the lungs showed a massive bilateral pulmonary haemorrhage (fig 2). No thrombi of the main pulmonary arteries were identified. The main airways were occluded by blood and clots (fig 2). The pulmonary veins were collapsed. Three main bronchial arteries (two 

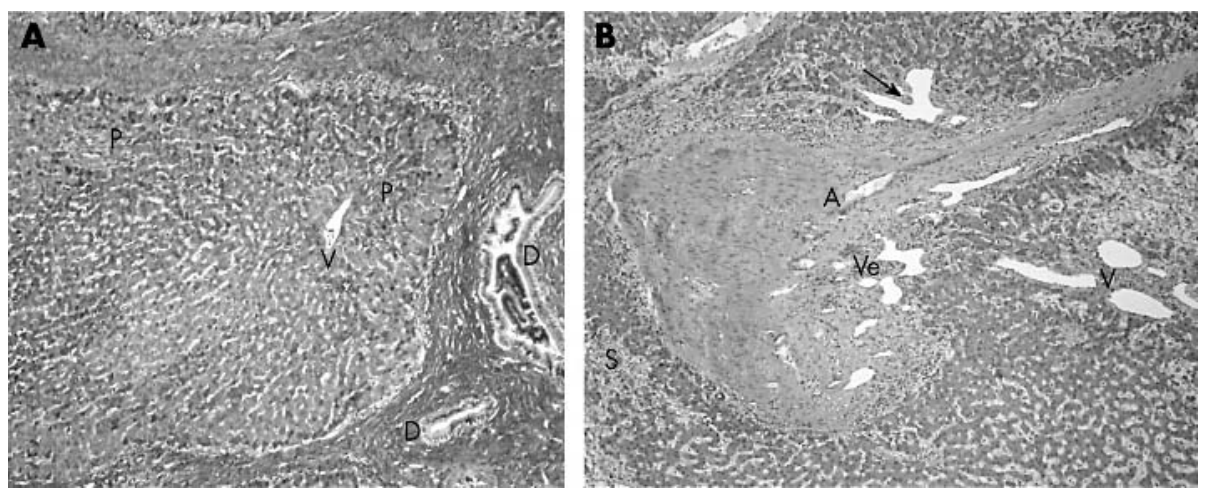

Figure 1 Native liver histology: general architecture. (A) Extremely large fibrous septa entrapping near normal "hepatic macrolobules" containing occasional portal tracts (P) and veins (V); large bile ducts (D) are irregularly embedded in the surrounding fibrous tissue. Chromotrope aniline blue method; original magnification, $x 40$. (B) Native liver histology: interesting features. Fibrous band containing small veins (Ve) and tangentially cut arterial vessels (A). Greatly dilated sinusoids (S) are found in the surrounding parenchyma. The black arrow points to an irregular, thin walled vascular space, directly abutting the hepatocyte cords. In addition, dilated vein-like vessels (V) are sparse among the hepatocyte cords. Haematoxylin and eosin stained; original magnification, $\times 40$.

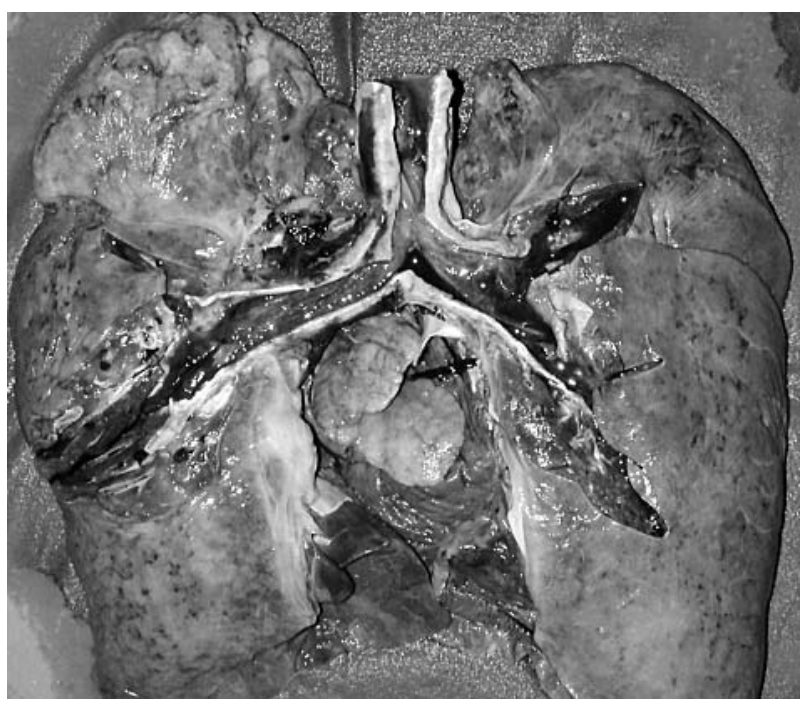

Figure 2 Lungs and tracheobronchial tree viewed from behind, after dissection of the pars membranacea of the trachea and of the main left and right bronchi, showing large blood clots occluding the airways. branches for the main right bronchus and one branch for the left main bronchus) were identified, and they were dilated and tortuous.

The main histopathological findings were:

- Massive bronchial haemorrhage with secondary alveolar flooding.

- The presence of a highly developed intrabronchial and peribronchial vascular plexus constituted by large telangiectatic vessels.

- An interesting lesion of the main pulmonary peripheral vessels was recognised: it was characterised by arteries showing either irregular eccentric intimal thickening, possibly related to previously organised thrombi or myointimal hyperplasia of sparse small sized arteries (fig $3)$. Interlobular veins were greatly dilated. These features were highly reminiscent of the lesions seen in arteriovenous malformations, or fistulas in other regions of the human body.

- The cut surface of both lungs was diffusely haemorrhagic and a specific site of the massive haemorrhage could not be identified.

The hepatic graft showed mild steatosis and very sparse mononuclear cell portal inflammatory infiltrates, with super-
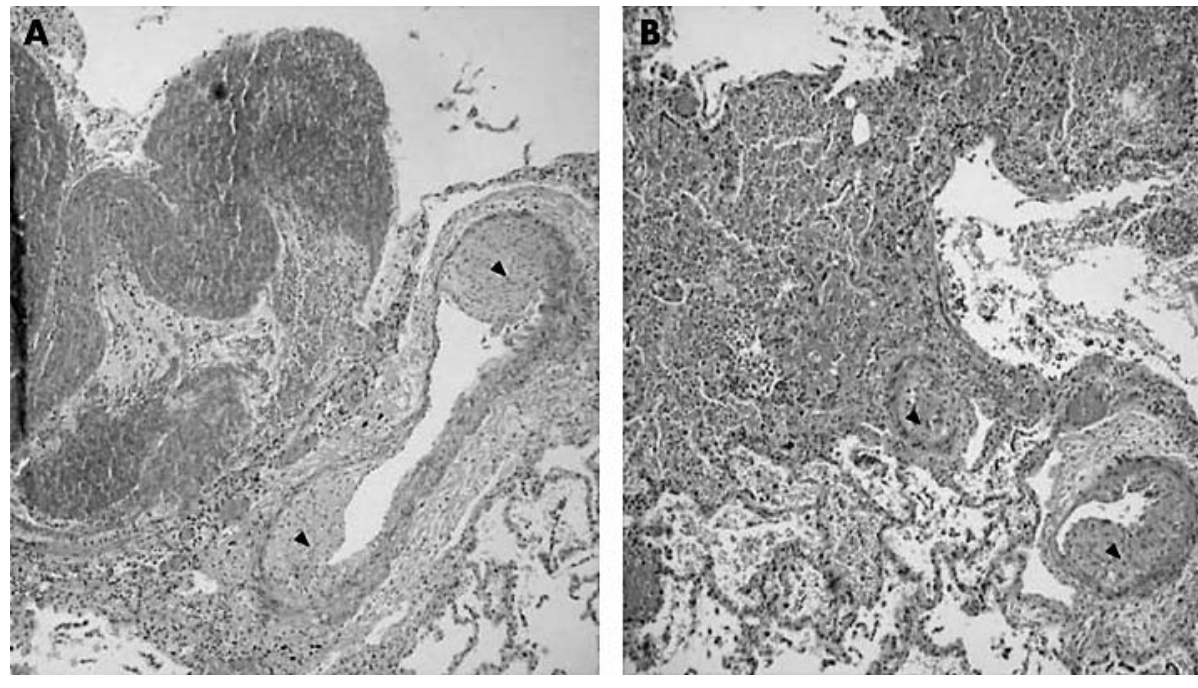

Figure 3 Massive alveolar haemorrhage and the main vascular lesions. (A) Eccentric intimal thickening of the dilated medium arteries (arrows; haematoxylin and eosin stained; original magnification, $\times 100)$ and $(B)$ myointimal hyperplasia of small sized pulmonary arteries (arrows; haematoxylin and eosin stained; original magnification, $\times 200$ ). 


\section{Take home messages}

- We report a 27 year old man with hereditary haemorrhagic telangiectasia who developed fatal progressive liver dysfunction after living related right lobe transplantation

- Pulmonary arteriography did not reveal arteriovenous malformation or abnormal intrapulmonary venous channels in this patient

- This case suggests that massive haemoptysis can develop even when arteriovenous malformations are undetectable by pulmonary arteriography

- It also questions the role and the appropriateness of living donor liver transplantation in high risk patients

imposed haemorrhagic lesions of the centrilobular areas, probably related to shock.

\section{DISCUSSION}

The clinical manifestations of HHT include vascular abnormalities of the nose, skin, lung, brain, and gastrointestinal tract. ${ }^{1}$

Pulmonary arteriovenous malformations affect $5-15 \%$ of patients with HHT. In some cases, standard chest $x$ ray, pulmonary angiography, or helical computed tomography can demonstrate a typical mass with enlarged arteries and veins or diffuse arteriovenous malformations.

In our patient, pulmonary angiography failed to demonstrate such lesions.

Liver involvement owing to the presence of multiple arteriovenous malformations or atypical cirrhosis is a rare but important manifestation of HHT. ${ }^{3} 7$

Our patient showed fibrosis, pseudocirrhosis, and highly dilated vein-like and arterial-like structures.

\section{"We are concerned about the role that liver transplanta-} tion could have played in the fatal outcome in our patient"

Liver transplantation for HHT with decompensated end stage liver disease and mild to moderate pulmonary hypertension has been reported. ${ }^{3} 68$

These patients are usually classified as UNOS 2a transplant recipients; in this group, the mortality rate from cadaveric donors is $18 \%,{ }^{69}$ compared with more than $50 \%$ from living related donors. ${ }^{10}$

In this setting, many transplant centres do not agree to perform living donor liver transplantation.
However, our patient had severe hypoxaemia, with a life expectancy of less than three months, and an unpredictable wait to receive a cadaveric liver.

In our patient, the cause of death was massive pulmonary haemorrhage from the rupture of one or many angiodysplastic bronchial lesions. This complication could not be predicted from the angiographic study, which was unable to detect arteriovenous malformations. The prevalence of pulmonary arteriovenous malformations among patients with HHT has been estimated to be $15-33 \% .^{11}$

Ference et al have reported the occurrence of pulmonary haemorrhage in $11(8 \%)$ of 143 patients with HHT not submitted to liver transplantation. ${ }^{2}$

Although diffuse pulmonary haemorrhage can appear during the natural history of HHT, we are concerned about the role that liver transplantation could have played in the fatal outcome in our patient.

\section{Authors' affiliations}

P Aseni, Department of General Surgery and Abdominal Organ Transplantation Niguarda Hospital, 20162 Milan, Italy

M Vertemati, Department of Human Anatomy, Faculty of Medicine, 20162 Milan, Italy

E Minola, E Bonacina, Department of Pathology Niguarda Hospital,

20162 Milan, Italy

Correspondence to: Dr M Vertemati, Via Mangiagalli 31 31, Milano

20130, Italy; maurizio.vertemati@unimi.it

\section{REFERENCES}

1 Guttmacher AE, Marchuk DA, White RI, Jr. Hereditary hemorrhagic telangiectasia. N Engl J Med 1995;333:918-24.

2 Ference BA, Shannon TM, White RI, et al. Life-threatening pulmonary hemorrhage with pulmonary arteriovenous malformations and hereditary hemorrhage telangiectasia Chest 1994; 106:1387-90.

3 Haitiiema T, Westermann TJ, Overtoom T, et al. Hereditary hemorrhagic telangiectasia (Osler-Weber-Rendu disease): new insights in pathogenesis, complications and treatment. Arch Int Med 1996:714-19.

4 Garcia-Tsao G, Korzenik JR, Young L, et al. Liver disease in patients with hereditary hemorrhagic telangiectasia. N Engl J Med 2000;343 931-6.

5 Reilly PJ, Nostrant TT. Clinical manifestations of hereditary hemorrhagic telangiectasia. Am J Gastroenterol 1984:79:363-7.

6 Boillot O, Bianco F, Mion F, et al. Liver transplantation resolves the hyperdinamic circulation in hereditary hemorrhagic telangiectasia with hepatic involvement. Gastroenterology 1999;1 16:187-92.

7 Martini GA. The liver in hereditary haemorrhagic telangiectasia: an inborn error of vascular structure with multiple manifestations: a reappraisal. Gut $1978 ; 19: 531-7$

8 Pfitzmann R, Heise M, Langrehr JM, et al. Liver transplantation for treatment of intrahepatic Osler's disease: first experiences. Transplantation 2001; 72:237-41

9 Kam I. Adult-adult right hepatic lobe living donor liver transplantation for status 2a patients: too little, too late. Liver Transpl 2002;8:347-9.

10 Testa G, Malago M, Nadalin S, et al. Right-liver living donor transplantation for decompensated end-stage liver disease. Liver Transp/ 2002;8:340-6.

11 Kjeldsen AD, Oxhoj $\mathrm{H}$, Andersen PE, et al. Pulmonary arteriovenous malformations: screening procedures and pulmonary angiography in patients with hereditary hemorrhagic telangiectasia. Chest 1999; 116:432-9. 